\title{
A spatial analysis of mental retardation of unknown cause and maternal residence during pregnancy
}

\author{
Huiling Zhen ${ }^{1}$, Andrew B. Lawson ${ }^{1}$, Suzanne McDermott ${ }^{2}$, Archana Pande Lamichhane ${ }^{1}$, \\ Marjorie Aelion ${ }^{3}$ \\ ${ }^{1}$ Department of Epidemiology \& Biostatistics; ${ }^{2}$ Department of Family and Preventive Medicine; and \\ ${ }^{3}$ Department of Environmental Health Sciences, University of South Carolina, USA
}

\begin{abstract}
Spatial analysis is useful for the identification of areas of elevated risk of adverse health outcomes and generation of hypotheses. Identification of clusters based on maternal residence during pregnancy provides an important tool to investigate risk exposures. However, even though mental retardation (MR) is a substantial public health problem, there are no previous analyses of spatial clustering of childhood MR using individual case data. In this paper, we examine the use of the Bayesian hierarchical modeling approach in the analysis of MR clustering. We used data from South Carolina Medicaid and birth certificates, in which address codes for each month of pregnancy are available. MR cases with unknown cause were identified in the study population. A Bayesian local likelihood cluster modeling technique was applied to compute the relative risk of MR and its corresponding P-value for each geo-coded location, and the P-value surface was contoured as a heat image to identify the MR clusters. The characteristics of the study population were analyzed using chi-square tests and the results confirm that clustering does occur for MR. The shapes of the identified MR clusters were found to be irregular and the observed MR rate in the identified MR cluster area was found to be double the rate for the larger South Carolina region. The descriptive analysis of study population characteristics showed that the children with MR were more likely to be male and had mothers who were older than 34 years at the time of birth as well as being African American, preterm and of low birth weight compared to children without MR.
\end{abstract}

Keywords: mental retardation, Medicaid, maternal, spatial, local likelihood, cluster, Bayesian.

\section{Introduction}

Spatial analysis is a very useful tool for the identification of areas of elevated risk of adverse health outcomes and generation of hypotheses. A spatial disease cluster can be defined as an area experiencing "unusually increased disease incidence rates". The term "hot spot" has been used to define a cluster if an "unusual" high rate is found (Lawson, 2006). Cluster detection methods used in spatial epidemiology have used both regional count data as

Corresponding author:

Andrew B. Lawson

Department of Epidemiology \& Biostatistics

Arnold School of Public Health

University of South Carolina

800 Sumter Street, Columbia, SC 292029, USA

Tel. +1 803777 6647; Fax +1 8037772524

E-mail: alawson@gwm.ec.edu well as case-control data, with controls selected from at-risk populations in order to estimate the relative risk or local rates. Most cluster analysis studies have used readily available software such as SaTScan (Kulldorff et al., 1997; Rainey et al., 2006; Cech et al., 2007; Wheeler, 2007) to identify disease clusters. However, SaTScan (www.satscan.org) has the limitation that it imposes a variety of constraints and produces limited output. First, a circular window is used and windows of varying size are examined to determine if the counts within these windows are "significantly in excess" compared to expected counts. The window with the most statistically significant cluster is identified as a primary cluster, and secondary clusters can also be reported. However, the output from the analysis is limited to primary and secondary clusters without any report of relative risk gradients which could display much 
more information about differentials of risk. The circular nature of the sampling window also limits the type of clusters that can be found; irregular clustering could be missed.

A recent example of a comparison of existing software-based methods was made by Wheeler (2007) who used individual-level childhood leukemia data to determine clusters based on two different methods: Kernel intensity estimation using $\mathrm{R}$ program (using Splancs) and scan statistics in SaTScan according to Kulldorff (1997). SaTScan is wellknown and the former approach is a weighting function used in non-parametric function estimation, and can be used to test for clustering and presence and location of local clusters. Bandwidth and distance are required by the Kernel intensity function, and need to be carefully chosen to get appropriate smoothing estimates. However, the findings regarding the identification of local clusters based on these two methods showed inconsistencies, calling for a more sensitive cluster analysis method.

Various spatial or space-time cluster analysis techniques have been used for cancer cluster detection. However, there are no previous analyses of spatial or spatio-temporal clustering of childhood mental retardation (MR) using individual case data even though MR is a substantial public health problem. For some time, environmental exposure to various kinds of influences have been suspected as a possible risk factor for some of the MR cases of unknown etiology but the methods employed to detect an association have been limited to conventional epidemiologic approaches.

MR is a developmental disability that becomes apparent in childhood or adolescence before the age of 18 . It is characterized by significant limitation in intelligence and daily living skills. In most cases, it persists throughout adulthood. MR occurs in 1.0$2.5 \%$ of the general population, and it has substantial public health implications.

Identification of MR clusters based on maternal residence during pregnancy is an important tool to investigate risky exposures. A recent Bayesian local likelihood cluster modeling techniques, which relies on the local likelihood and a "lasso" parameter, can be employed to identify MR clusters. By using this approach, the posterior relative risk for each geocoded maternal address is computed with the corresponding significant P-value surface, which can be contoured as a heat image to identify the MR clusters. Bayesian hierarchical modeling is a very useful tool to identify MR clusters. It allows flexible cluster forms, the incorporation of covariates, and also sensitivity analysis of assumptions. The local likelihood models use a "lasso" parameter, which defines the size of an area within which cases are accumulated. Thus, it produces a continuous risk map where risk gradients are apparent, and also the shapes of the clusters identified by this approach are not restricted to circles as in other methods (such as SaTScan).

In this paper, we examine the use of Bayesian hierarchical modeling to the analysis of MR clustering in South Carolina, USA, using maternal address codes for each month of gestation. The purpose of the study is to demonstrate whether clustering occurs for MR, and that irregular risk areas can be identified by this technique.

\section{Background with respect to $M R$}

MR affects 6 million American children under the age of 14 years (Shea, 2006). According to the Metropolitan Atlanta Developmental Disabilities Surveillance Program (MADDSP), the average annual prevalence of MR from 1991-1994 among 3-10 year-old children was 9.7 per 1,000 children. The Healthy People 2010 Objectives for the Nation include as Objective 16-14 "Reduce the occurrence of developmental disabilities". The target for MR is 124 per 10,000 for 8 year-old, and was established in 1985 based on the Metropolitan Atlanta Developmental Disabilities Surveillance System (MADDS). The average lifetime cost for one person with mental retardation is estimated at 1,014,000 US dollars (CDC, 2004).

MR has important public health implications for at least four reasons:

(i) its relatively high prevalence of 1-2.5 percent in 
most populations;

(ii) large contribution to years of productive life lost since its onset is in childhood with life-long disability as a result;

(iii) substantial socio-economic implications since disability is related to unemployment and public financing for services and health care; and

(iv) 50 percent of the cases have unknown cause (McDermott et al., 2007).

The most widely used definition of MR in the United States is from the American Association on Mental Retardation (AAMR), renamed as the American Association on Intellectual and Developmental Disabilities (AAIDD) in 2006. According to AAIDD, this affection is a disability that occurs before age 18 and is characterized by significant limitations in intellectual functioning and adaptive behaviour as expressed in conceptual, social and practical adaptive skills. It is diagnosed through the use of standardized tests of intelligence and adaptive behaviour. MR is generally thought to be present if an individual has an IQ test score of approximately 70 or below and a significant deficit in at least one area of adaptive behaviour (AAMR, 2002).

South Carolina is one of the very few states that have linked birth certificate data with administrative data from Medicaid inpatient and outpatient maternal and infant records. Even fewer states have linked these data to the care provided during pregnancy. No state, except South Carolina, has recorded address codes for each month of pregnancy that can be linked to the Medicaid and birth certificate data. Finally, half of the births in this state are insured by Medicaid which offers the unique opportunity to look at spacetime clustering of MR cases among the high-risk Medicaid-insured population using individual case/control data by gestational months of potential exposure. Due to the ability to link data, the clusters of MR in specific locations and in time can be explored to investigate associations between environmental exposure and MR controlling for maternal conditions during pregnancy, infant characteristics, and early childhood conditions. In what follows, we have employed this local likelihood clustering methodology to identify MR clusters in South Carolina, using maternal address codes for each month of gestation. We distorted the shape of the actual map to be a rectangle, as shown in Figures $2 \mathrm{a}-\mathrm{j}$, since the locations of risk are confidential following an agreement with the Medicaid agency. The actual code for replicating the method is available through correspondence with the corresponding author.

\section{Materials and methods}

The present study is a retrospective cohort study which included 22,429 maternal-child pairs, and child Medicaid inpatient and outpatient data during childhood. Maternal and child Medicaid data and birth certificates records were linked for women who were pregnant during the period January 1, 1996 through December 31, 2001. Addresses were obtained for each month of pregnancy, and geocoded to determine the monthly location of the mothers. Ten gestational months were used instead of nine since pregnancy can extend beyond 40 weeks of gestation. Although the first and tenth month had a smaller proportion of mothers, we included all pregnancies through birth of the infant. Women included in the first month of pregnancy usually had another child covered by Medicaid or were in a family covered by Medicaid. Child health records covering the period between birth and the year 2007 were also obtained.

Most of the women entered the Medicaid program in their second to fourth gestational months. Mothers entered the dataset throughout pregnancy while other mothers dropped out. Entry was based on obtaining Medicaid eligibility and drop-outs resulted from moving away, pregnancy loss, and change in eligibility status. Thus, during each month of pregnancy, we had a different denominator. The Medicaid reimbursement files for the pregnant mothers, birth certificate data, hospital and outpatient care for both the mother and the infant, and child health records were merged. As a result, we had maternal prenatal files and child health records for 
5-10 years following birth to identify codes for MR in the Medicaid record.

\section{MR case definitions}

The identification of MR cases in this analysis included two important steps. The strategy was designed to identify all cases of MR, and then exclude those that had a known genetic, infectious, injury or alcohol-related cause. We focussed on the cases with unknown cause since this is the group for whom an environmental exposure could be a risk factor. First, we identified a list of known causes of MR and their ICD9 codes using multiple sources (McDermott et al., 2007). We excluded 969 babies with a known cause of MR/developmental disability (DD). The excluded infants had one of the following known causes of MR/DD: trisomy 13, 16-18, other chromosomal aberrations, Prader-Willi syndrome, Rett's syndrome, phenylketonuria, fragile X syndrome, postnatal injury, prenatal rubella, meningitis, encephalitis, and fetal alcohol syndrome. The second step involved identifying infants and children with ICD 9 code 317, 318, or 319 in the Medicaid inpatient and outpatient records. Through this process we identified 792 cases of MR (3.5\%) (Fig. 1).

\section{Cluster detection}

Recently, a novel approach for the detection of spatial clusters was proposed for both count data in small areas and case event data at residential addresses (Hossain and Lawson, 2005; Lawson, 2006). This Bayesian local likelihood cluster modeling technique relies on the definition of a likelihood which is a function of a "lasso" parameter to identify the clusters. This parameter defines the size of an area within which cases are accumulated. Both cases of disease and controls can be accumulated in the "lasso". In situation where binomial data are available (such as births with and without MR), we simply count the number of MR cases within the "lasso" and the number of births without MR (control cases). The likelihood used is a local likelihood based on the "lasso" found for each site. The "lassos" can and do overlap. Local likelihood methods are designed to provide estimates of areas of excess risk across the continuous disease-risk surface of the map. When the algorithm is implemented, the posterior distribution of "lassos" is sampled over a large number of iterations and the posterior average "lasso" for each area is obtained. As a function of this "lasso", the relative risk is also obtained. The local relative risk is important for cluster detection as it can show where an excess of cases is to be found. We assumed that our clusters are essentially "hot spots" or areas of the map which show unusually high risk. The posterior average relative risk was computed, and the "significance" of the local risk elevation was assessed by the estimation of an exceedence probability. This probability is defined as $p_{i}=\operatorname{Pr}\left(R R_{i}>1\right)$ where $\mathrm{RR}_{\mathrm{i}}$ is the local relative risk at the $i$ th site. This is computed from the posterior sample of relative risks determined for each site. To comply with the usual criteria for significance tests, we examined $q_{i}=1-p_{i}$ for local areas with $q_{i}<0.05$ or 0.01 . The risk in these areas can be considered as unusual or significantly elevated. Note that our model provides a description of the spatial variation of risk on the map over all areas, and we do not need to adjust for multiple testing, unlike other methods such as SaTScan.

The advantages of this method are that: (i) it produces a continuous risk map where gradients of risk are apparent as well as clusters, (ii) it does not limit the clusters to circular shapes, and (iii) it automatically allows the incorporation of covariates within a full likelihood formulation. The final output from the local likelihood sampler is in the form of a P-value surface. This surface can then be contoured or displayed as a heat image or perspective plot, and areas of highly excessive risk of any shape will be represented by areas below the 0.05 or 0.01 contour levels.

\section{Statistical analyses}

The characteristics of the Medicaid study population, with and without MR, were analyzed using 


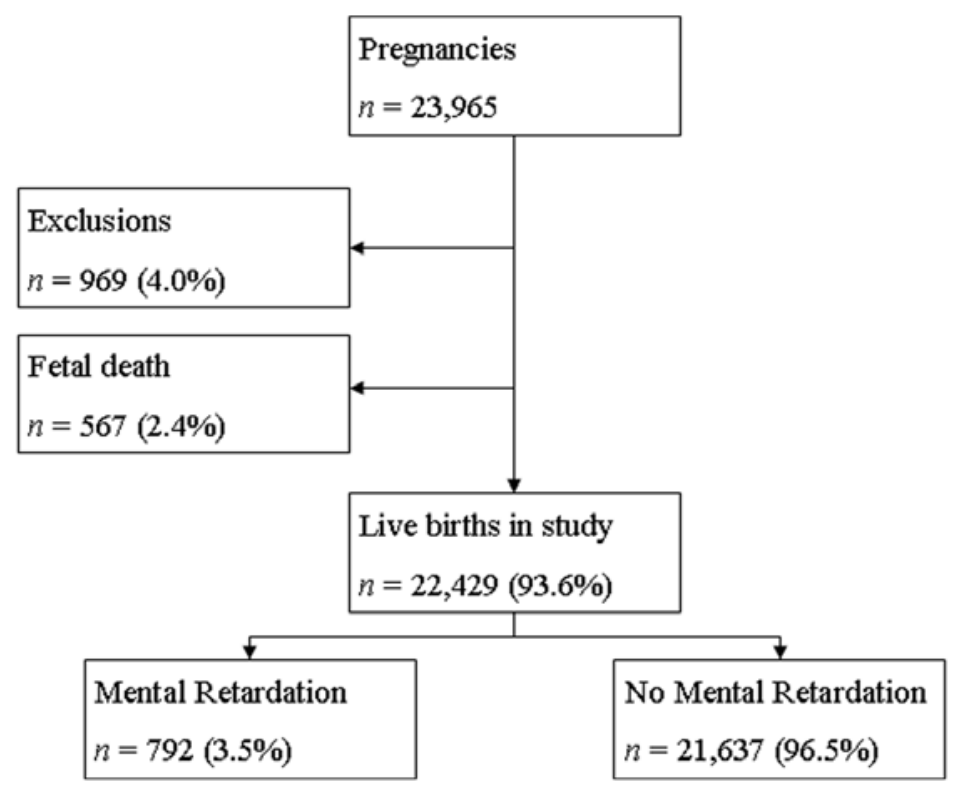

Fig. 1. Flow chart of study population.

chi-square tests to determine if there were statistically significant differences in the proportions within the considered categories (Table 1).

The analysis of the residential data was based on case and control data to avoid unstable regional rates caused by small numbers of observed cases and small population counts (Devine et al., 1996). The process of cluster analysis started with the removal of pregnancies outside the study area using a point-in-polygon (PIP) function in FORTRAN, which checked the geo-code of each pregnancy within South Carolina, and removed the pregnancy if the geo-coded addresses were located outside the study area. Cluster analysis was performed using data files for each gestational month (all-year data combined by gestational month) for MR cases. The relative risk of $\mathrm{MR}$ and $\mathrm{P}$-values were estimated for each geo-coded location according to the Bayesian local likelihood cluster modeling techniques (Lawson, 2006). A heat image map "pixellated" with colours corresponding to the P-values was created using $\mathrm{R}$ program, and contour lines were plotted at different P-values based on smoothing techniques (MBA R package).
In order to identify the MR clusters in each gestational month, data for the years 1996-2001 were grouped together. We created 10 monthly MR contour graphs. For a better understanding of the MR rate in each month, point maps of cases and controls were plotted separately side-by-side for each month using $\mathrm{R}$ program.

Next, we divided the study area into a grid mesh based on sample size, and each region contained around 5,000 observations. In each grid cell, the posterior expected relative risk of MR and the corresponding $\mathrm{P}$-value were estimated using MCMC local likelihood cluster models. The P-values were determined based on each divided region and were adjusted to the global P-value by multiplying the ratio of overall $\mathrm{MR}$ rates over the divided-region $\mathrm{MR}$ rate. Then, contour lines were plotted based on global p-values in each geo-coded location, and MR clusters were identified from the contour graph. If clusters were found close to the boundary of adjacent regions, new regions containing the boundary of the adjacent divided regions were checked to see if edge effects existed. To check edge effects, P-values were calculated by averaging the 
P-value from the divided region and the P-value from the checked region. Then, the P-value was adjusted by the ratio of the overall MR rate over the divided-region MR rate.

For the first gestational month, the area was divided into five small regions, and 3-edge regions were checked for edge effect. For the second gestational month, the area was divided into nine small regions. As the number of gestational months increased, the sample size increased dramatically due to the ongoing entry into Medicaid throughout pregnancy. The area was divided into much smaller regions to have appropriate sample size and more boundary edges were checked for potential edge effects. For example, in the seventh month, the area had to be divided into 15 regions to have appropriate sample size. At the point where there were too many divided regions and edge-effect checks to keep the results stable and reliable, we switched our analysis to be based on count data.

Finally, count data analysis (the number of observations in each small cell) was conducted to identify clusters. Data conversion was performed using ArcGIS. The first step identified appropriate geographic coordinate systems/units for the data, and the boundary file formed a converted polygon for the area. A total of 2,032 grid cells were created within the boundary, with each grid cell measuring 3,500 $\mathrm{m} \times 3,00 \mathrm{~m}$. The centroid of each grid cell was extracted, and numbers of MR cases and controls in each grid cell were counted. The cumulative relative risk of $\mathrm{MR}$, and corresponding P-values were calculated for the centroid location of each grid cell based on an MCMC local likelihood sampling technique, performed in FORTRAN program. Then, a "pixellated" heat image map with colours corresponding to the P-values was created using $\mathrm{R}$ program. The locations of grid lines corresponded to the centroid location of each grid cell. Contour lines were plotted at different P-value cut-off based on smoothing techniques. MR clusters were identified based on contour graphs for all 10 gestational months.

\section{Results}

Some of the known characteristics associated with MR among the study population are shown in Table 1. The table shows that children with MR were more likely to be male and had mothers who were older than 34 years at the time of birth. They were also predominantly African Americans, very preterm ( $<28$ weeks gestation) and of lower birth weight compared to the group without MR.

For the 10 gestational months, we obtained separat exceedence maps for the MR risk. They are displayed in Figure $2(\mathrm{a}-\mathrm{j})$. Based on the P-values, an MR cluster can be identified around the Midwestern part of the contour surface maps, which is the area of $x(0.125,0.5)$ and $y(0.45,0.65)$ based on the coordinates system of the contour surface maps. This cluster is consistently present at a similar position in all 10 gestational months' maps, with only some movement from month to month. This unstable location may be partially explained by the different influence of gestational month on the child's MR development. The shape of the identified MR cluster is irregular, instead of circular. Also, the Pvalue gradient of the excess risk of this MR cluster can be determined based on the contour surface map, which could provide more information about the differentials of MR risk, and is very useful for finding a MR cluster area where a range of outcomes is available.

The cluster area identified in the mid-western part of the contour surface maps is confirmed by the observed MR rate, as shown in Table 2 and Figure 3. The observed Medicaid MR rate in the identified cluster area is $5 \% \sim 8.8 \%$, while the general Medicaid MR rate in South Carolina is around $3 \% \sim 4 \%$. For all 10 gestational months, the observed MR rate in the cluster area is higher than the general observed rate in South Carolina. In most gestational months, the observed MR rate in the cluster area is twice the rate in South Carolina, especially for gestational month 10 . Those excess MR rates further confirmed the presence of the clusters identified by the local likelihood approach. 
Table 1. Characteristics of the study population across the study area in South Carolina, 1996-2001.

\begin{tabular}{|c|c|c|c|c|}
\hline \multicolumn{2}{|c|}{ Characteristics of mothers and children } & \multirow{2}{*}{$\frac{\text { No. MR (\%) }}{21312(98.8)}$} & \multirow{2}{*}{$\frac{\text { No. MR (\%) }}{773(98.1)}$} & \multirow{2}{*}{$\begin{array}{r}\text { P-value } \\
0.086\end{array}$} \\
\hline Alcohol $^{1}$ & No & & & \\
\hline & Yes & $262(1.2)$ & $15(1.9)$ & \\
\hline \multirow[t]{2}{*}{ Baby sex } & Boy & $10962(50.7)$ & $516(65.1)$ & \multirow[t]{2}{*}{$<0.001$} \\
\hline & Girl & $10674(49.3)$ & $276(34.8)$ & \\
\hline Mother age & $<18$ & $2130(9.8)$ & $65(8.2)$ & \multirow[t]{3}{*}{$<0.001$} \\
\hline \multirow[t]{2}{*}{ (years) } & $18-34$ & $18542(85.7)$ & $654(82.6)$ & \\
\hline & $>34$ & $965(4.5)$ & $73(9.2)$ & \\
\hline \multirow[t]{3}{*}{ Mother race } & White & $8286(38.4)$ & $198(25.0)$ & \multirow[t]{3}{*}{$<0.001$} \\
\hline & African American & $13042(60.4)$ & $585(73.9)$ & \\
\hline & Other & $265(1.2)$ & $9(1.1)$ & \\
\hline \multirow[t]{3}{*}{ Weeks gestation } & $<28$ & $120(0.6)$ & $67(8.9)$ & \multirow[t]{3}{*}{$<0.001$} \\
\hline & $28-36$ & $2522(12.1)$ & $157(20.8)$ & \\
\hline & $>36$ & $18122(87.3)$ & $530(70.3)$ & \\
\hline Birth weight & $<1500$ & $240(1.1)$ & $118(14.9)$ & \multirow[t]{3}{*}{$<0.001$} \\
\hline \multirow[t]{2}{*}{ (g) } & $1500-2500$ & $1831(8.5)$ & $125(15.8)$ & \\
\hline & $>2500$ & $19560(90.4)$ & $547(69.2)$ & \\
\hline \multirow[t]{4}{*}{ Parity } & 0 & $9438(43.6)$ & $316(39.9)$ & \multirow[t]{4}{*}{0.080} \\
\hline & 1 & $6448(29.8)$ & $242(30.6)$ & \\
\hline & 2 & 3451 (15.9) & $131(16.5)$ & \\
\hline & $3+$ & $2300(10.6)$ & $103(13.0)$ & \\
\hline
\end{tabular}

${ }^{1}$ Alcohol use reported on birth certificate.

In addition to the cluster identified in the midwestern part of the contour surface maps, several other MR clusters can also be identified in South Carolina, such as the one around $\mathrm{x}(1.5,1.7)$ and $\mathrm{y}$ $(0.3,0.5)$ in the contour maps of most gestational months. The shape and the size of the identified MR clusters vary across South Carolina, and the P-value gradient of the excess risk of MR can be determined.

To validate the MR diagnoses, we linked the Medicaid child files with the file from the Department of Disabilities and Special Needs (DDSN) and the Department of Education (DOE) to identify gold standard cases of MR. We identified all children enrolled in DDSN for early intervention services and children receiving special education in school for MR (equivalent to EMH = educable mentally handicapped or $\mathrm{TMH}=$ trainable mentally handicapped). Since South Carolina does not have a private school for children with MR we have good follow-up for these children. There are private schools for children without MR and $14.1 \%$ of children in the state attend private schools, according to the 2000 census. The proportion of children who attend public schools is higher for those who are African-American compared to Caucasian, and also for those whose families have comparatively lower incomes. Thus, for our Medicaid study group a high proportion will be enrolled in public schools. For the portion of the state analyzed in this study, approximately $20 \%$ of the Medicaid MR cases were identified as gold standard cases of MR.

\section{Discussion}

The identification of MR clusters based on maternal residence during pregnancy represents an important step forward in elucidating causes of this condition. Despite the advances in genetic and radiologic diagnostics, the proportion of $\mathrm{MR}$ cases with unknown cause has been approximately 40-50\% 
a) Month 1

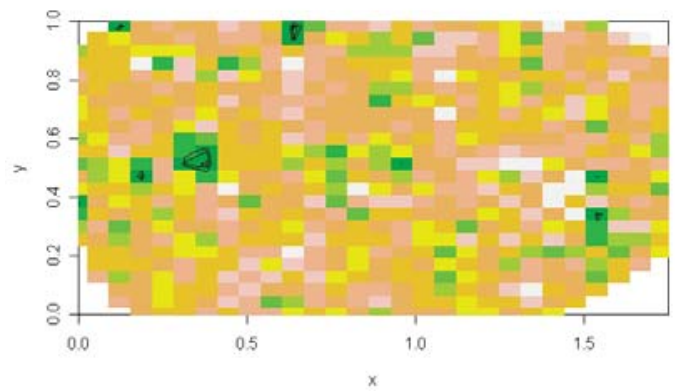

b) Month 2

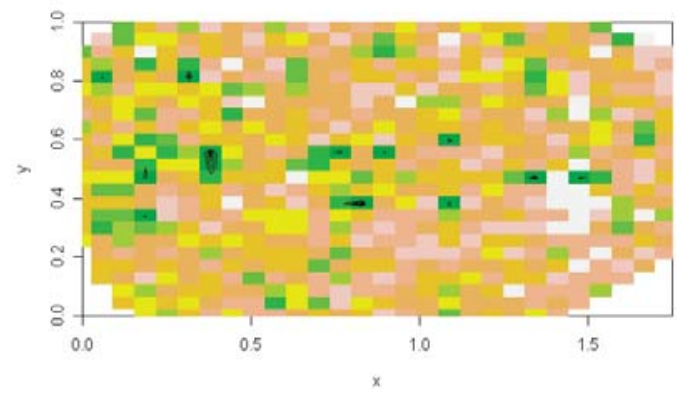

c) Month 3

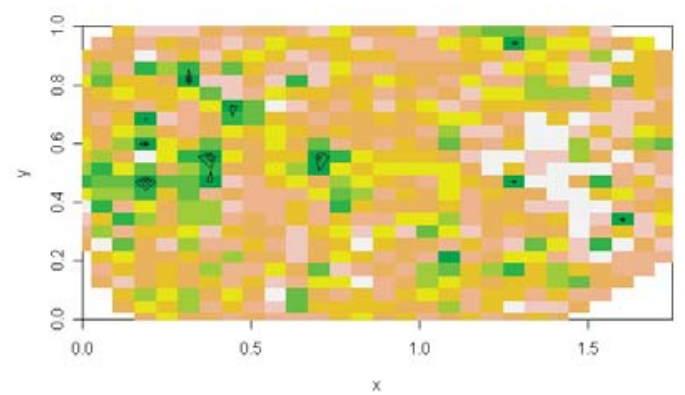

d) Month 4

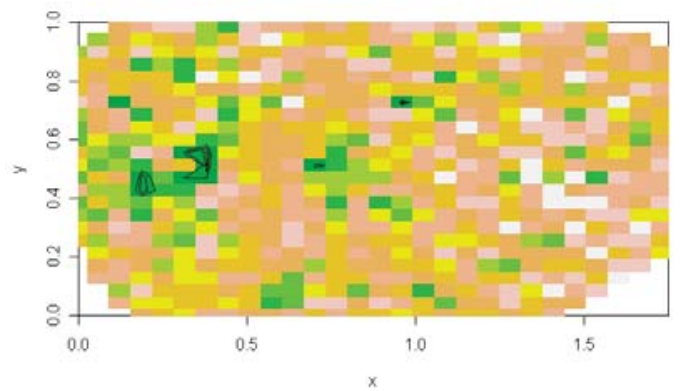

e) Month 5

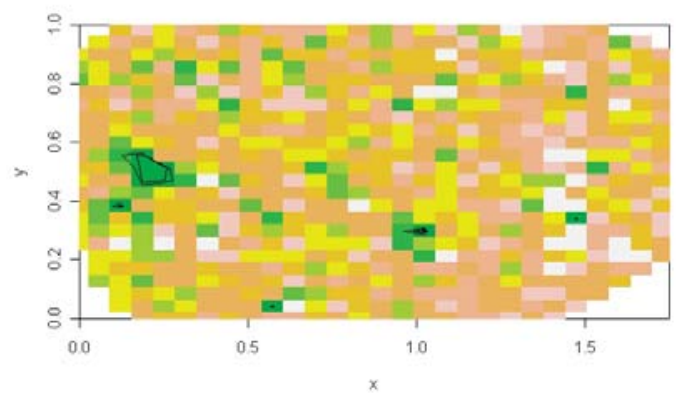

f) Month 6

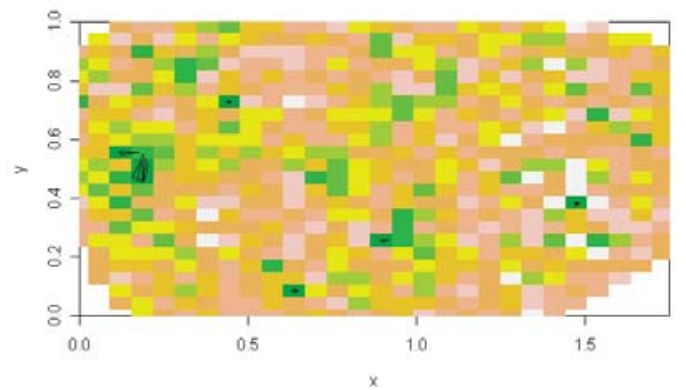

g) Month 7

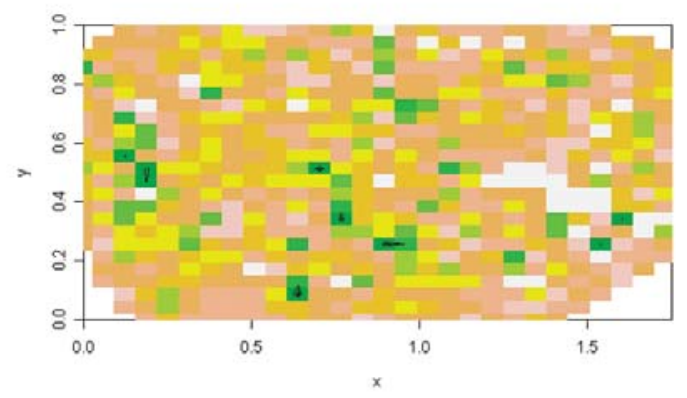

h) Month 8

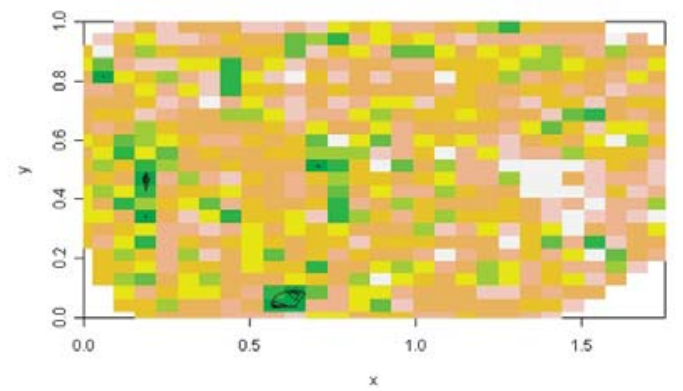

Fig. 2 (a-j). Heat image and contour map of P-values for excess risk of MR based on local likelihood exceedence probability, months 1-10 (contours drawn at P-values $0.01,0.05$, and 0.1); 0.5 units in $\mathrm{x}$-axis indicate 30 miles, 0.2 units in y-axis indicate 14 miles. 
i) Month 9

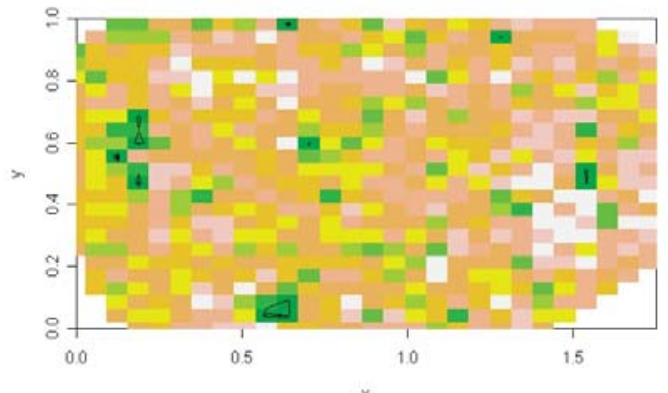

j) Month 10

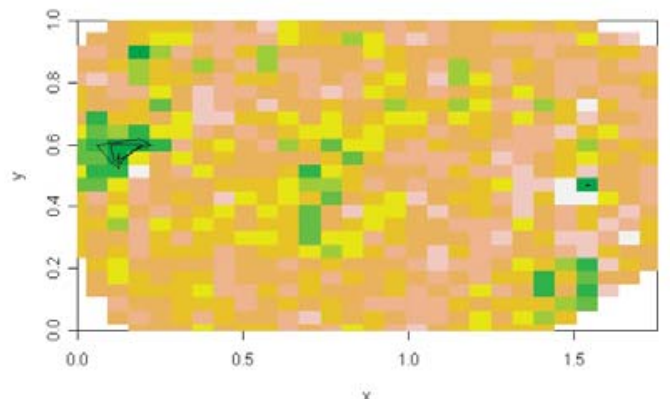

Fig. $2(a-j)$. Continued.

Table 2. MR rates in the cluster area and across the study area in South Carolina by gestational month, 1996-2001.

\begin{tabular}{lllllll} 
& \multicolumn{3}{c}{ General MR rate in South Carolina } & \multicolumn{3}{c}{ MR rate in cluster area } \\
\hline Months & MR cases & Total & Rate per 100 & MR cases & Total & Rate per 100 \\
\hline Month 1 & 1287 & 30126 & 4.3 & 11 & 185 & 5.9 \\
Month 2 & 2213 & 54029 & 4.1 & 19 & 273 & 7.0 \\
Month 3 & 2813 & 70991 & 4.0 & 27 & 337 & 8.0 \\
Month 4 & 3169 & 80749 & 3.9 & 31 & 366 & 8.5 \\
Month 5 & 3331 & 86235 & 3.9 & 30 & 388 & 7.7 \\
Month 6 & 3386 & 89436 & 3.8 & 30 & 395 & 7.6 \\
Month 7 & 3225 & 90194 & 3.6 & 29 & 408 & 7.1 \\
Month 8 & 2887 & 84396 & 3.4 & 26 & 370 & 7.0 \\
Month 9 & 2091 & 65491 & 3.2 & 15 & 281 & 5.3 \\
Month 10 & 523 & 16442 & 3.8 & 7 & 79 & 8.9 \\
\hline
\end{tabular}

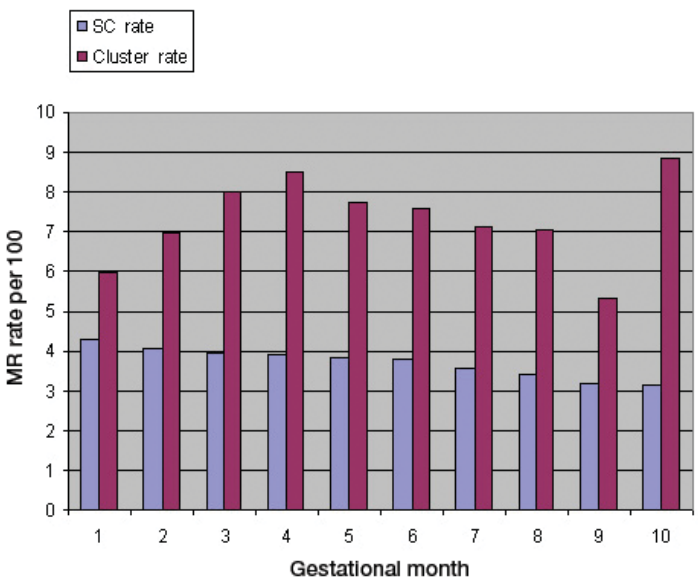

Fig. 3. MR rates in the cluster area and across the entire study area in South Carolina by gestational month, 1996-2001. over the last decades. The identification of causes of MR has been complicated by the recognition that some anatomical changes are only the proximal cause of MR. Thus, some of the actual insults that are formed at the time of conception, or during pregnancy, remain illusive. It is conceivable that exposure to environmental chemicals plays a role, and the identification of clusters will help researchers to explore hazardous sites in the vicinity of these clusters.

This study was only possible because we had access to data for each month of maternal residence for women insured by the Medicaid program. Half of all births in South Carolina are insured by Medicaid, and these children represent the group at highest risk of MR due to factors associated with poverty. 
Some issues related to the validity of MR diagnosis that plague other studies are minimized in this investigation. The use of address codes to identify clusters is not based on residence when the diagnosis of MR was made but on maternal residence during pregnancy. Thus, the bias of some physician's use of the ICD9 codes for the diagnosis of MR would have impacted studies that rely on child addresses, but is much less an issue when maternal pregnancy addresses are used.

A limitation of the study reported here is that, as yet, we have not examined the association between clusters in MR outcome and associated explanatory variables. Table 1 shows that the common risk factors identified in the literature are also associated with MR in our cohort. The cluster analysis cannot rule out the possibility that the relationship between maternal residence is operating through one of these risk factors.

There is movement of mothers during pregnancy. In our study cohort, $22 \%$ moved once, $2 \%$ moved twice and $0.1 \%$ moved more than twice during pregnancy. In addition, there is movement out of Medicaid throughout childhood as the income eligibility changes for families. During pregnancy, Medicaid is the most inclusive with families eligible up to 1.85 times the federal poverty level. Since also children with a disability qualify for Medicaid, we expect there will be higher retention of children with MR compared to children without a disability.

In conclusion, this investigation allowed us to associate maternal location during each month of gestation with MR. The identification of irregularshaped clusters for this lifelong disability with childhood onset is an important advance. The observed MR rate in the cluster area is double the rate for the study area in South Carolina. The next step will involve development of hypotheses how environmental exposures, e.g. particular chemicals, are related to MR.

\section{References}

American Association on Mental Retardation (AAMR), 2002. Mental retardation: definition, classification, and systems of supports, 10th Edition, Washington, DC, USA.

Cech I, Burau KD, Walston J, 2007. Spatial distribution of orofacial cleft defect births in Harris county, Texas, 1990 to 1994, and historical evidence of the presence of low-level radioactivity in tap water. South Med J 100, 560-569.

Centers for Disease Control and Prevention (CDC), 2004. Economic costs associated with mental retardation, cerebral palsy, hearing loss, and vision impairment - United States, 2003. MMWR 53, 57-59.

Devine OJ, Louis TA, Halloran ME, 1996. Identifying areas with elevated disease incidence rates using empirical Bayes estimators. Geogr Anal 28, 187-199.

Hossain M, Lawson AB, 2005. Local likelihood disease clustering: development and evaluation. Environ Ecol Stat 12, 259-273.

Kulldorff M, Feuer EJ, Miller BA, Freedman MS, 1997. Breast cancer clusters in Northeast United States: a geographic analysis. Am J Epidemiol 146, 161-170.

Lawson AB, 2006. Disease cluster detection: a critique and a Bayesian proposal. Stat Med 25, 897-916.

McDermott, S, Durkin, MS, Schupf, N, Stein, Z, 2007. Epidemiology and Etiology of Mental Retardation: In: Handbook of Intellectual and Developmental Disabilities, JW Jacobson, JA Mulick, J Rojahn (eds). Springer Press, New York, USA.

Metropolitan Atlanta Developmental Disabilities Surveillance Program http://www.cdc.gov/ncbddd/dd/maddsp.htm\#prev.

Rainey JJ, Omenah D, Sumba PO, Moormann AM, Rochford R, Wilson ML, 2006. Spatial clustering of endemic Burkitt's lymphoma in high-risk regions of Kenya. Epidemiology 120, 121-127.

Shea SE, 2006. Mental retardation in children ages 6 to 16 . Semin Pediatr Neurol 13, 262-270.

Wheeler DC, 2007. A comparison of spatial clustering and cluster detection techniques for childhood leukemia incidence in Ohio, 1996-2003. Int J Health Geogr 27, 13. 